\title{
Editorial
}

\section{Autism: From genetics to biomarkers}

\author{
Irina Voineagu \\ RIKEN Omics Science Center, Yokohama, Kanagawa, 230-0045, Japan \\ Tel.: +81 45503 9222; Fax: +81 45503 9236; E-mail: irinavoineagu@gmail.com
}

The development of biomarkers for psychiatric disorders is particularly challenging, given the frequent clinical heterogeneity, and the subjective nature of symptoms which requires finely tuned quantification methods for accurate diagnosis. Yet the more clinically heterogeneous a disease, the more valuable becomes an objective measure of disease state or severity. While identifying biological markers for psychiatric disorders has thus far remained out of reach, groundbreaking progress in DNA sequencing technologies and the completed human genome sequence have set the foundation for a novel perspective on clinical care, which also brings promising new approaches for biomarker discovery. The aim of genomic medicine [1] is to take advantage of the wealth of information encoded in an individual's genome regarding their disease risk and potential responses to therapy. For diseases having significant contribution of genetic factors, the possibility to quantify genetic variation with enough depth is expected to lead to the development of effective markers of disease risk.

This special issue of Disease Markers is dedicated to autism spectrum disorders (ASD) and discusses the current understanding of ASD genetics, as well as the possibilities of translating genetic research toward biomarker development in ASD.

ASD are a spectrum of neurodevelopmental conditions characterized by language deficits, dysfunctions of social-reciprocal interactions and repetitiverestrictive behaviors. The clinical manifestations of autism are highly variable, both between individuals, and along an individual's developmental trajectory. Although some individuals with ASD are highly functional, many are severely impaired and require permanent care. The significant level of impairment combined with the fact that no specific therapy is yet available for ASD, make ASD a devastating illness for patients and families, and a heavy financial burden for the healthcare system. The most effective intervention for ASD has proven to be early behavioral therapy [2]. Thus the identification of biological markers for ASD, allowing very early detection, even before the onset of symptoms, would be of tremendous value At the same time, one of the most well established characteristics of ASD is it's high heritability, and significant research efforts have been geared toward understanding the genetic basis of autism. The field of ASD genetic research is still far from fully elucidating the mechanisms that govern ASD heritability, but the last two decades have undoubtedly brought about remarkable progress. Currently it is believed that both common genetic variation and rare DNA sequence variants contribute to the ASD genetic susceptibility [3], and that the relative contribution of common and rare alleles is variable among ASD cases.

In the first article of this issue, "Mutant mouse models of autism spectrum disorders", Yuri Bozzi and colleagues review the phenotypic characteristics of currently available mouse models of ASD as well as the contribution of mouse models toward the development of pharmacological therapy for ASD. Of particular importance for dissecting out the cellular and molecular mechanisms of ASD are several genes that have been identified as causes of genetic syndromes with a high incidence of ASD (FMRl, the gene mutated in fragile X syndrome, TSC1/TSC2, the genes responsible for tuberous sclerosis and $N F 1$, the gene implicated in neurofibromatosis are just a few examples). Despite the 
fact that mutations in each of these genes cause ASD in a minority (less than $2 \%$ ) of cases, they represent valuable targets for animal models, in which the effect of specific mutations can be investigated at both cellular and behavioral levels, in a controlled genetic background. The authors highlight the diversity of phenotypes observed in mouse models of ASD, likely reflecting the genetic and phenotypic heterogeneity of the disease.

While behavioral paradigms are well developed for the study of ASD-like social deficits and repetitive behaviors in rodents, these model organisms lack an appropriate behavioral equivalent of human language. In the article "A songbird animal model for dissecting the genetic bases of autism spectrum disorder" Carmen Panaitof discusses the role of the songbird as an experimental model system for investigating the genetic basis of human language and the language impairments associated with ASD.

To further dissect out the mechanisms of language deficits in ASD, Michael Bowers and Genevieve Konopka review recently emerging evidence for the role of the FOXP family of transcription factors in human language and ASD. FOXP2 was the first gene to be directly implicated in genetic regulation of language in humans, through the identification of FOXP2 mutations in a family affected by speech impairment. Although genetic association studies have not yet conclusively demonstrated the involvement of FOXP2 in ASD, several of the FOXP2 downstream targets are ASD susceptibility genes. The authors discuss the possible roles of signaling pathways regulated by $F O X P 2$, as well as two additional members of the FOXP family, FOXP1 and FOXP4, in neurodevelopment, language and ASD.

The review by Alka Saxena, Dave Tang and Piero Carninci focuses on the functional roles of $M E C P 2$, the gene mutated in the majority of cases of Rett syndrome, one of the autism spectrum disorders. In their manuscript "piRNAs warrant investigation in Rett syndrome: An omics perspective", the authors put forward the interesting hypothesis that piRNAs, a class of small non-coding RNAs, might mediate the transcriptional effects of $M E C P 2$. The authors also discuss a potential role for this class of non-coding RNAs as molecular biomarkers for Rett syndrome.

In the review concluding this special issue, "Subphenotype-dependent disease markers for diagnosis and personalized treatment of autism spectrum disorders", Valerie Hu discusses the current progress toward identifying ASD biomarkers based on genome-wide data, including gene expression, non-coding RNAs and epigenetic modifications. This article also highlights the importance of defining ASD clinical subphenotypes in order to increase the likelihood of biomarker identification.

In summary, the articles in this series give an overview of genetic models of ASD, discuss several key emerging concepts in understanding the molecular basis of ASD (the role of FOXP transcription factors and the potential roles of noncoding RNAs) and outline the current stage of biomarker development with a particular focus on genomic data.

It is exciting times for genetic research and although the phenotypic and genetic heterogeneity of ASD often seem to be a daunting conundrum, well defined diagnostic criteria, larger cohort sizes for genetic studies and integrative approaches of genomic and epigenomic data already delineate a promising avenue for elucidating the mechanisms of ASD.

\section{References}

[1] Boone, P.M., W. Wiszniewski, and J.R. Lupski, Genomic medicine and neurological disease. Hum Genet, 2011. 130(1): pp. 103-21.

[2] LeBlanc, L.A. and J.M. Gillis, Behavioral interventions for children with autism spectrum disorders. Pediatr Clin North Am, 2012. 59(1): pp. 147-64, xi-xii.

[3] State, M.W. and P. Levitt, The conundrums of understanding genetic risks for autism spectrum disorders. Nat Neurosci, 2011. 14(12): pp. 1499-506. 


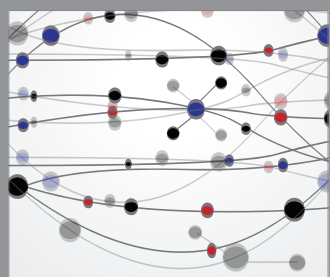

The Scientific World Journal
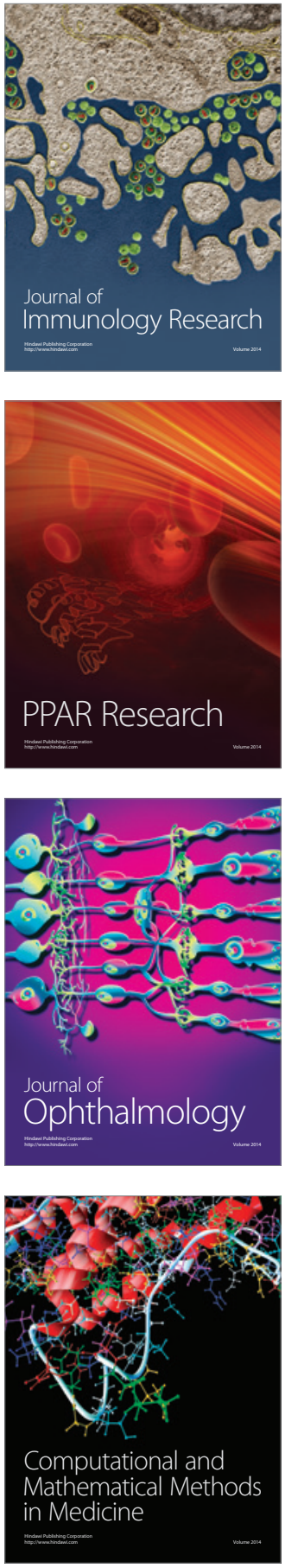

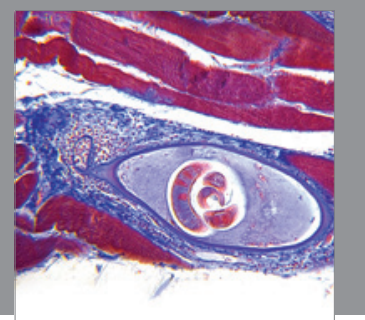

Gastroenterology

Research and Practice
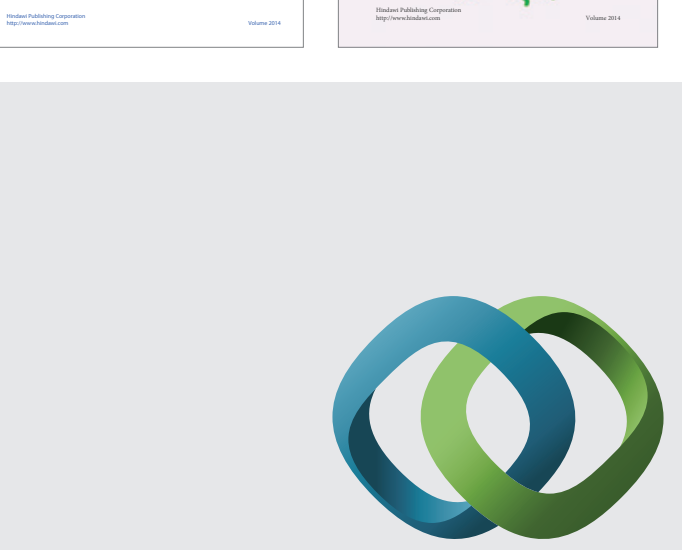

\section{Hindawi}

Submit your manuscripts at

http://www.hindawi.com
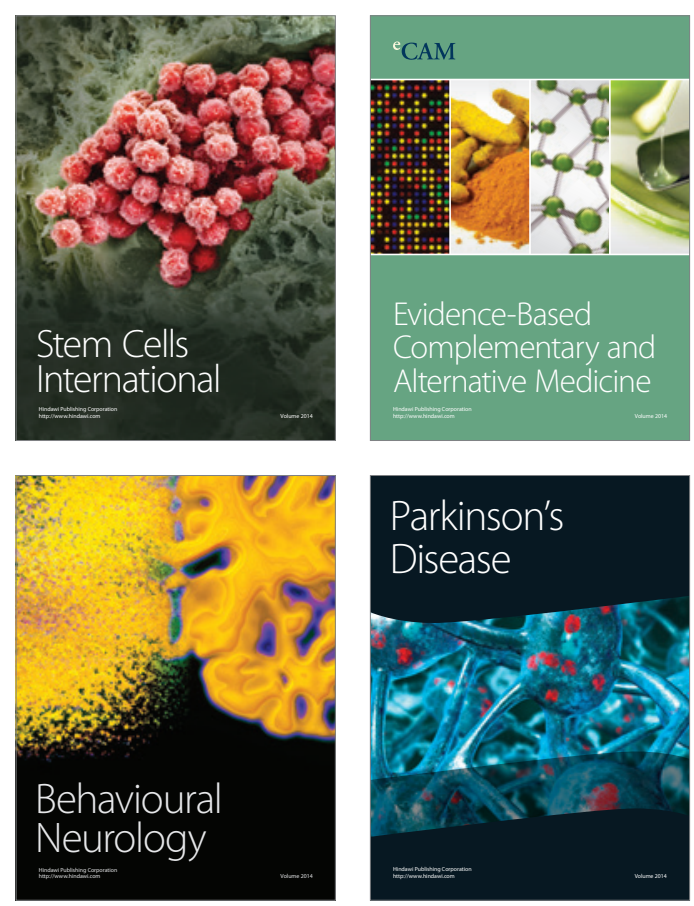

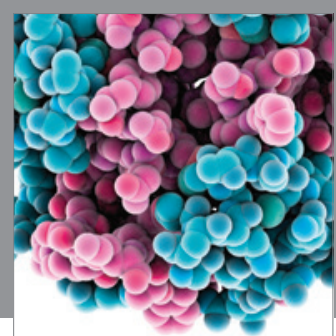

Journal of
Diabetes Research

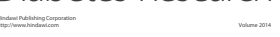

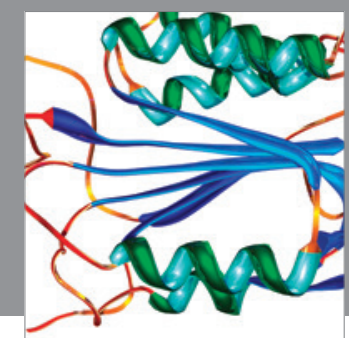

Disease Markers
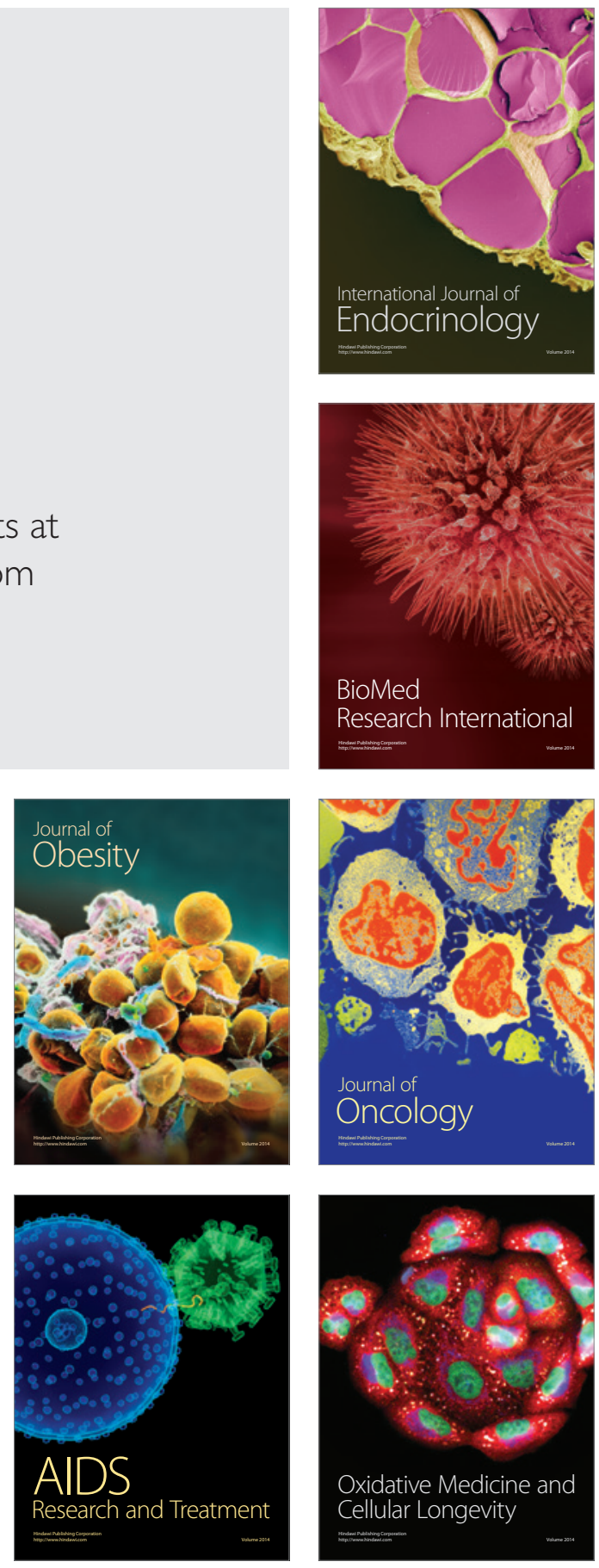